\title{
gs \\ Structure maps for hcp metals from first-principles calculations
}

\author{
Ohad Levy, ${ }^{1,2}$ Gus L. W. Hart, ${ }^{3}$ and Stefano Curtarolo ${ }^{1,4, *}$ \\ ${ }^{1}$ Department of Mechanical Engineering and Materials Science and Department of Physics, Duke University, \\ Durham, North Carolina 27708, USA \\ ${ }^{2}$ Department of Physics, NRCN, P.O. Box 9001, Beer-Sheva, Israel \\ ${ }^{3}$ Department of Physics and Astronomy, Brigham Young University, Provo, Utah 84602, USA \\ ${ }^{4}$ Department of Materials and Interfaces, Weizmann Institute of Science, Rehovot 76100, Israel
}

(Received 14 February 2010; published 10 May 2010)

\begin{abstract}
The ability to predict the existence and crystal type of ordered structures of materials from their components is a major challenge of current materials research. Empirical methods use experimental data to construct structure maps and make predictions based on clustering of simple physical parameters. Their usefulness depends on the availability of reliable data over the entire parameter space. Recent development of highthroughput methods opens the possibility to enhance these empirical structure maps by $a b$ initio calculations in regions of the parameter space where the experimental evidence is lacking or not well characterized. In this paper we construct enhanced maps for the binary alloys of hcp metals, where the experimental data leaves large regions of poorly characterized systems believed to be phase separating. In these enhanced maps, the clusters of noncompound-forming systems are much smaller than indicated by the empirical results alone.
\end{abstract}

DOI: $10.1103 /$ PhysRevB.81.174106

PACS number(s): 61.50.Ah, 61.66.Dk, 81.30.Bx

\section{INTRODUCTION}

Predicting the stable structures of alloys from their constituents is a major challenge of current materials research. ${ }^{1,2}$ The traditional approach to this problem is to extract trends from systems for which experimental data is available and apply them to uncharacterized systems. ${ }^{3}$ These rules usually depend on simple parameters, e.g., atomic number, atomic radius, electronegativity, ionization energy, melting temperature or enthalpy, etc. Several well-known methods include the Hume-Rothery rules, ${ }^{4}$ the Miedema formation enthalpy method, ${ }^{5}$ the Zunger pseudopotential radii maps, ${ }^{6}$ and Pettifor maps. ${ }^{7,8}$

These methods produce results largely consistent with existing experimental data and have helped direct a few successful searches for unobserved compounds. ${ }^{9}$ However, they offer a limited response to the challenge of identifying new compounds because they rely on the existence of consistent and reliable information for systems spanning most of the relevant parameter space. In many cases, reliable information is missing in a large portion of the parameter space-less than $50 \%$ of the binary systems have been satisfactorily characterized. ${ }^{10}$ This leaves considerable gaps in the empirical structure maps and reduces their predictive ability.

High-throughput (HT) calculations of material properties based on density-functional theory have been developed for theoretically guided material discovery and design. ${ }^{11-17}$ In this approach the phase stability landscape of alloys is explored by calculating the formation enthalpies of a large number of possible structures, identifying the minima at various component concentrations. A minimum-free-energy convex hull is constructed from these minimum-energy structures. These calculations give insights into trends in alloy properties and indicate possible existence of hitherto unobserved compounds. Recent advancements in this method, by efficiently covering extensive lists of candidate structure types, ${ }^{18-21}$ make it possible to complement sparse experi- mental data with $a b$ initio total-energy assessments. This development was envisioned by Pettifor ${ }^{9}$ a few years ago and here we present its realization for the binary systems of the hcp metals. We apply the HT approach to a comprehensive screening of the 105 intermetallic binary systems of the hcp metals. These systems have been generally less extensively studied than the cubic (fcc and bcc) metals, both experimentally and theoretically. The experimental data is particularly sparse for a large group (46) of binary intermetallics believed to be phase separating (see Fig. 1). Ab initio calculations on these systems are scarce compared to bcc and fcc systems. Cluster expansion ${ }^{22,23}$ studies of hcp systems ${ }^{24-27}$ are also relatively rare compared to cubic systems, probably due to the inherent additional computational difficulties in dealing with a lattice having a basis of symmetrically identical atoms [a general cluster expansion code for arbitrary parent lattices was not available until 2002 (Ref. 28)].

\section{METHODS}

Computations of low-temperature stability of the hop metallic binary systems were carried out using the HT framework AFLOW (Refs. 14 and 29) and ab initio calculations of the energies with the VASP software ${ }^{30}$ with projector augmented waves pseudopotentials ${ }^{31}$ and the exchangecorrelation functionals parameterized by Perdew, Burke, and Ernzerhof ${ }^{32}$ for the generalized gradient approximation. The energies were calculated at zero temperature and pressure with spin polarization and without zero-point motion or lattice vibrations. All crystal structures were fully relaxed (cell volume and shape and the basis atom coordinates inside the cell). Numerical convergence to about $1 \mathrm{meV} /$ atom was ensured by a high-energy cutoff $(30 \%$ higher than the maximum cutoff of both potentials) and dense $6000 \mathrm{k}$-point Monkhorst-Pack meshes. ${ }^{33}$

For each system, we calculated the energies of the reported crystal structures ${ }^{34,35}$ and approximately 226 addi- 


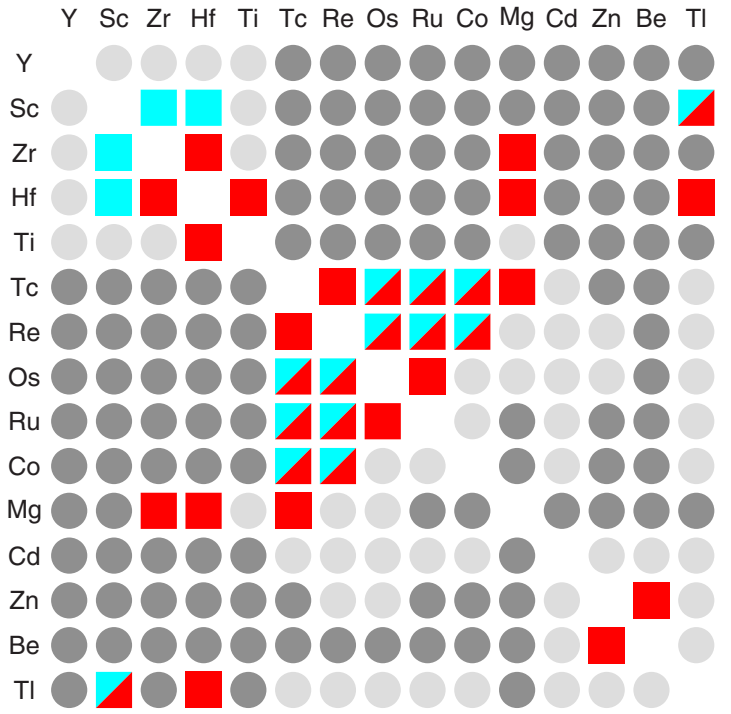

FIG. 1. (Color online) Compound formation or phase separation in binary systems of the hep metals, ordered by the Pettifor $\chi$ scale. Circles denote agreement of the empirical data and HT calculations on a database of $\sim 226$ structures for phase-separating (light gray) and compound-forming (dark gray) systems. Squares denote HT prediction of stable ordered structures in systems reported as phase separating: blue (light gray), red (dark gray), or mixed for hcp, non-hcp, or both types of predicted structures, respectively.

tional structures in the AFLOW database. ${ }^{29}$ These include the 176 configurations described in Ref. 14, all the symmetrically distinct hcp-, bcc-, fcc-based superstructures ${ }^{36,37}$ with up to four atoms per cell, and the prototypes A5, A6, A7, A8, A9, A11, B20, C36, D5 $19, \mathrm{Al}_{2} \mathrm{Zr}_{4}, \mathrm{Al}_{3} \mathrm{Zr}_{2}, \mathrm{BiHf}_{2}^{\star},{ }^{19} \mathrm{CdTi}$, $\mathrm{CuPt}_{7}, \mathrm{Cu}_{3} \mathrm{Ti}_{2}, \mathrm{Ga}_{2} \mathrm{Hf}, \mathrm{Ga}_{4} \mathrm{Ni}, \mathrm{Ga}_{3} \mathrm{Pt}_{5}, \mathrm{Ga}_{4} \mathrm{Ti}_{5}, \mathrm{Hf}_{3} \mathrm{Sc}^{\star}, 19$ $\mathrm{Hf}_{5} \mathrm{Sc}^{\star},{ }^{19} \mathrm{Hf}_{5} \mathrm{~Pb}^{\star},{ }^{19} \mathrm{HfPd}_{5}^{\star},{ }^{19} \mathrm{Hf}_{2} \mathrm{Tl}^{\star},{ }^{19} \mathrm{Hg}_{2} \mathrm{Pt}$, ITl, InTh, LiB-MS1/2, ${ }^{38,39} \mathrm{MoTi}^{\star},{ }^{14} \mathrm{Mo}_{3} \mathrm{Ti}^{\star},{ }^{14} \mathrm{NbNi}_{8}, \mathrm{NiTi}_{2}$, SeTl, and $\mathrm{V}_{4} \mathrm{Zn}_{5}$. In Ref. 14, it was shown that the probability of reproducing the correct ground state, if well defined and not ambiguous, is $\eta_{c}^{\star} \sim 96.7 \%$ ["reliability of the method," Eq. (3)]. There is no guarantee that the true ground states of a system will be found among the common experimentally observed structures or among small-unit-cell derivative structures. However, even if it is impossible to rule out the existence of an unexpected ground state, this protocol (searching many enumerated derivative structures and exhaustively exploring experimentally reported structures) is expected to give a reasonable balance between high-throughput speed and scientific accuracy to determine miscibility (or lack thereof) in these alloys.

\section{RESULTS AND DISCUSSION}

Of the 105 hcp-hcp metallic binary systems, 46 were previously reported as phase separating. ${ }^{34,35}$ Our calculations show that 18 of those are actually compound forming at low temperatures. Most of the predicted compounds are not hcpderived structures. These results are summarized in Fig. 1, where all the hcp metals are ordered according to Pettifor's ${ }^{7,8}$ chemical scale $\chi$. The symbols in each intersecting cell indicate the phase-separating or compound-forming nature of the binary alloys. Circles denote cases in which the HT calculation results match the experimental data for phase-separating (light gray) and compound-forming (dark gray) systems. Alloys where discrepancies are found, i.e., alloys reported as phase separating but predicted to have stable ordered structures, are denoted by squares, blue (light gray) — if the predicted structures are hcp derived, or red (dark gray) - if they are not hcp based. Squares with both colors indicate a prediction of both types of ground states in the same system. No reverse discrepancies were found, i.e., no cases were found where systems reported to be compound forming were predicted to be phase separating at low temperatures.

Most of the 46 reported phase-separating systems are grouped in this map into three clusters: ten systems in the upper left corner, six in the lower right corner, and 23 in a large cluster in the center. The last metal in the map, thallium, has ten phase-separating systems, seven of which are not in these three clusters. The $a b$ initio results predict four compound-forming systems grouped in the center of the upper left cluster, which is reduced to just six systems. The central cluster is also reduced to just 12 systems. In particular, $\mathrm{Mg}-\mathrm{Zr}$ and $\mathrm{Hf}-\mathrm{Mg}$, which form an extended branch of this cluster are predicted to be compound forming. The new smaller cluster is thus better localized in the chemical scale space. Alternative Mendeleev number assignments are sometimes suggested (e.g., see Ref. 10). One such suggestion places $\mathrm{Mg}$ and $\mathrm{Be}$ adjacent to each other before Sc and Y. The map in Fig. 1 indicates that the ordering according to the Pettifor $\chi$ scale locates them more appropriately at the lower part of the scale. A few of these alternative arrangements also place Sc before Y, whereas our calculations indicate that the $\chi$ scale is again more appropriate, since it groups together the predicted compound-forming systems in the upper left cluster. Two isolated phase-separating systems, Sc-Tl and Hf-Tl, are predicted to be compound forming, so it now seems that $\mathrm{Tl}$ should be more appropriately placed between $\mathrm{Cd}$ and $\mathrm{Zn}$. The calculation results thus complement the empirical Pettifor map and group all the phase-separating systems into three small well-defined clusters.

Figures 2-4 show the resultant structure maps $\left(\chi_{A}, \chi_{B}\right)$ for the binary $A B, A B_{2}$, and $A B_{3}$ compounds. The ordered structures are represented by their Strukturbericht designation or prototype. A circle indicates agreement of the empirical data and the HT calculations. Cases of disagreement are indicated by rectangles. The upper and lower entries in each cell give the empirical data and the HT result, respectively. In cases where two different structures are reported in the literature or found degenerate in the calculations, both are indicated as upper or lower entries. A dash (-) indicates the absence of a stable structure. Unidentified suspected structures are denoted by a question mark (?). Some of the predicted phases (marked by an asterisk *) have structures for which no prototype is known and no Strukturbericht designation has been given. These unreported prototypes are described in Table I.

Systems reported as phase separating but predicted by our HT results to be compound forming are highlighted in red (dark gray) rectangles. In systems reported to be compound forming, discrepancies between observed and calculated structures are highlighted in blue (light gray). In these cases, the energy difference between the structures or between the 


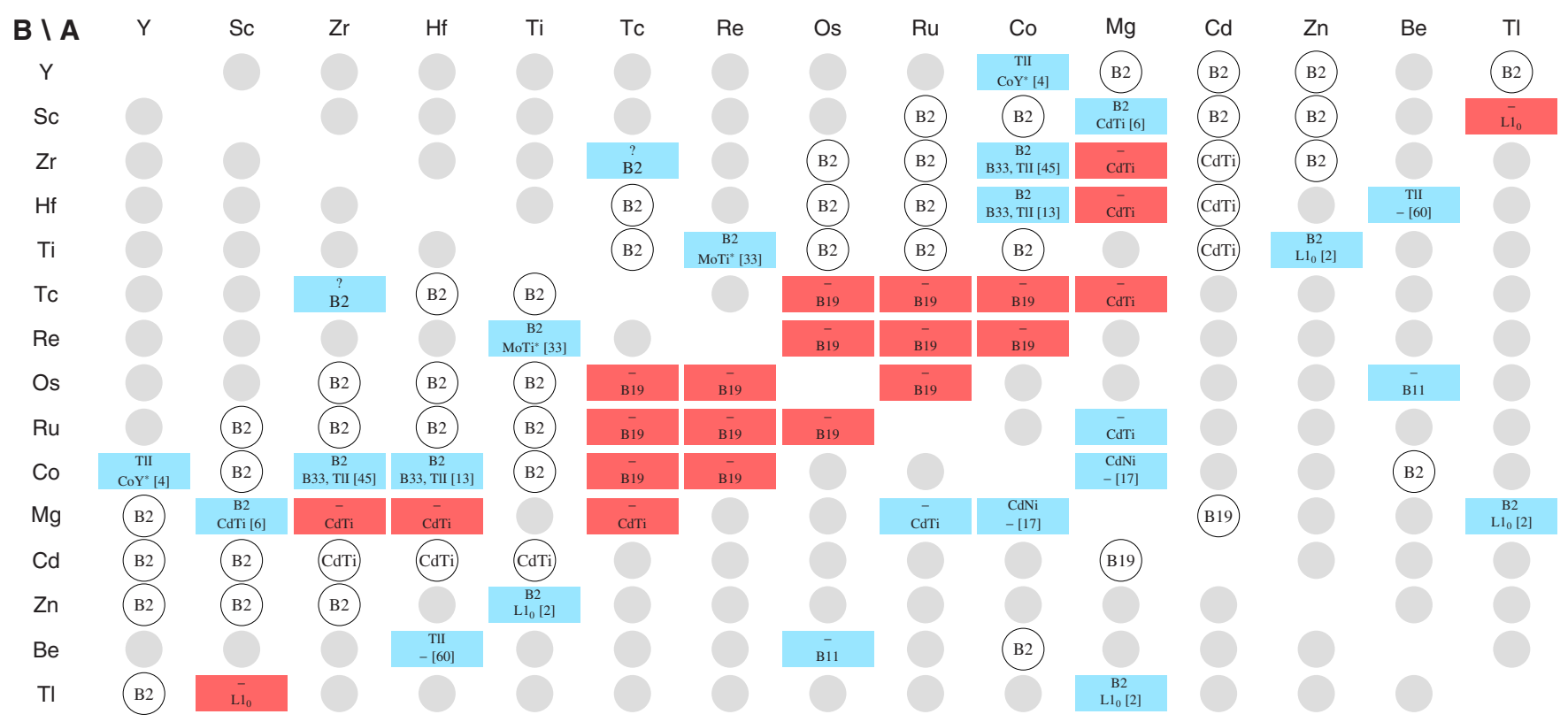

FIG. 2. (Color online) The $A B$ structure map for hcp alloys from empirical data (upper entry in each cell) and HT calculations (lower entry). Circles with a single entry indicate agreement between experiment and calculation. Predicted ordered structures in reported phaseseparating systems are highlighted in red (dark gray). Discrepancies between observed and calculated structures are highlighted in blue (light gray). Suspected compounds with unknown prototypes are denoted by a question mark. Unreported prototypes are marked by $\star$ and described in Table I. The energy difference between reported and calculated structures or between the reported structure (unstable in the calculation) and a two-phase tie line is indicated in square parentheses.

reported structure (unstable in the calculation) and the twophase tie line is indicated in square parentheses.

Most of the predicted compounds in the $A B$ map are concentrated in a large cluster at the center, in the largest gap of the empirical map. Seven of them form a cluster of B19 structures. Three $\mathrm{Mg}$ compounds of the CdTi prototype neighbor the reported group of $\mathrm{Cd}$ compounds with the same structure. The main feature of the empirical $A B$ map is a large cluster of B2 compounds. The ab initio calculations largely preserve this cluster and the separation between it and the adjacent group of CdTi compounds. Discrepancies between reported and predicted structures appear mostly at

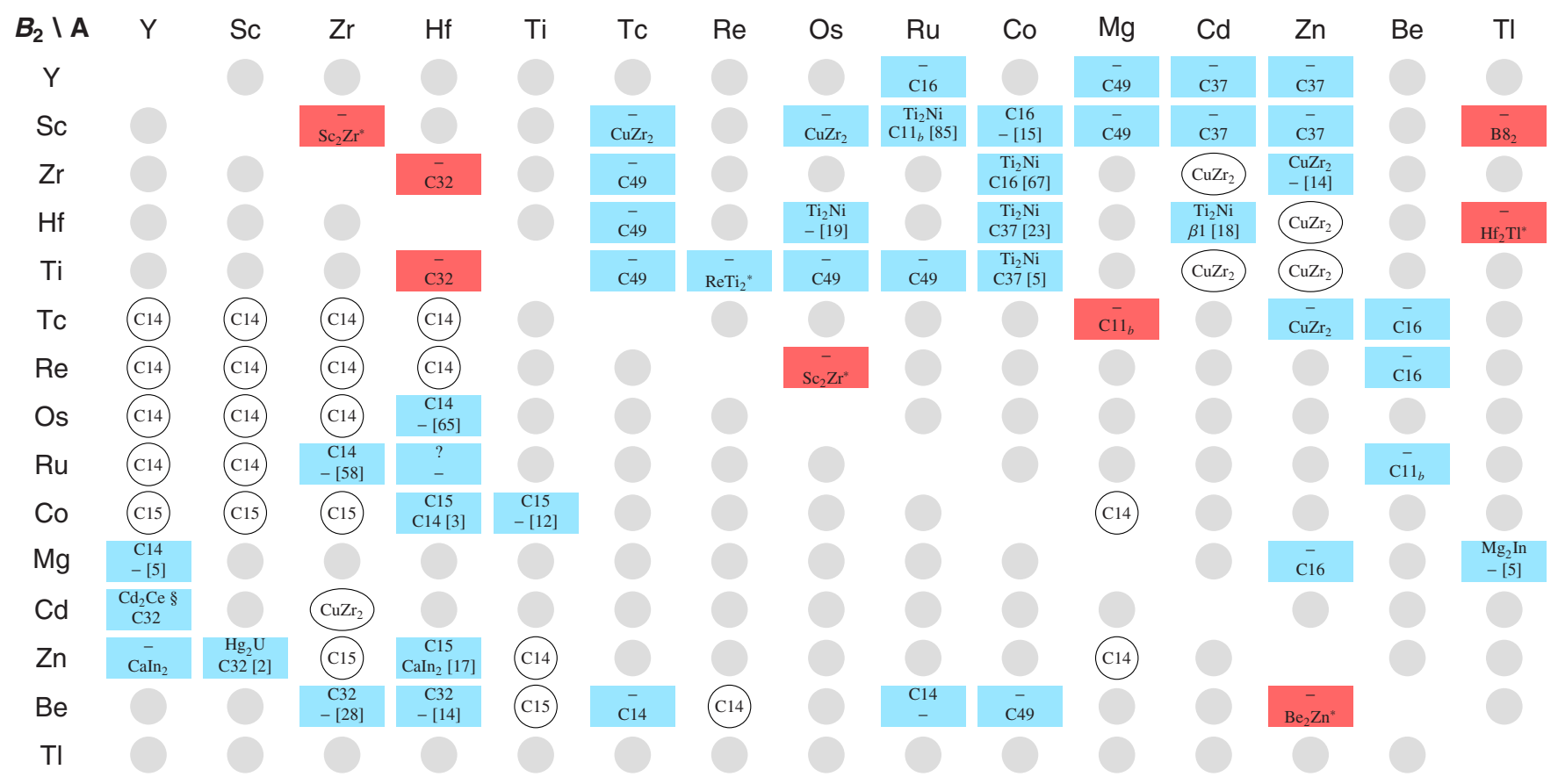

FIG. 3. (Color online) The $A B_{2}$ structure map for hcp alloys from empirical data and HT calculations. Notations are as in Fig. 2. $\S$ indicates that the reported prototype relaxed to the predicted one in the calculation. 


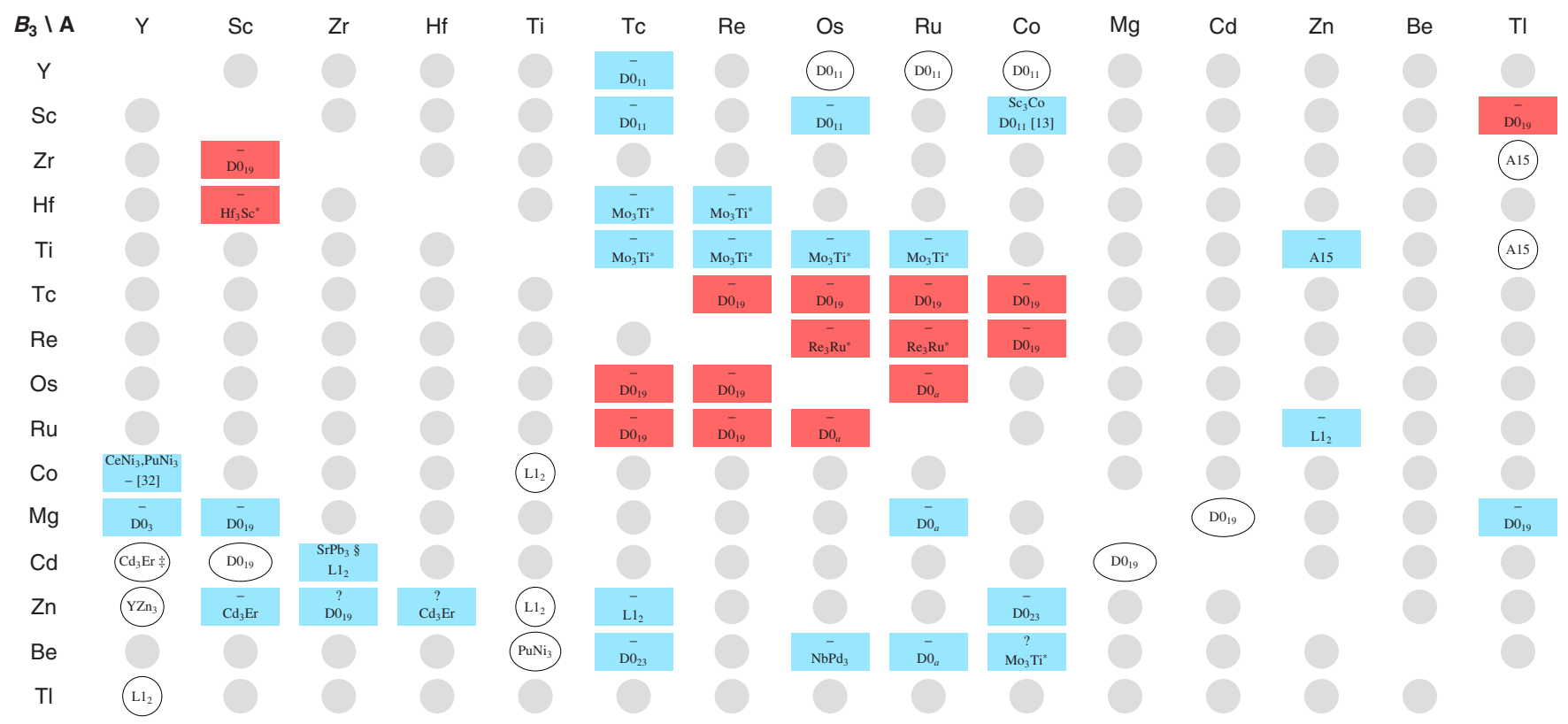

FIG. 4. (Color online) The $A B_{3}$ structure map for hep alloys from empirical data and HT calculations. Notations are as in Figs. 2 and 3. \$ In the case of, $\mathrm{YCd}_{3}$, the high-throughput results find that the $\mathrm{Cd}_{3} \mathrm{Er}$ structure and $\mathrm{D}_{19}$ have the essentially same energy.

the margins of these clusters. For example, the MgSc compound, reported as B2, is predicted to switch to the adjacent CdTi cluster at low temperatures. An unidentified TcZr compound (denoted by a question mark) is predicted to belong to the B2 group and an unreported MgRu compound is predicted to belong to the CdTi cluster. Most of the energy differences between reported and predicted structures are small. Relatively large differences are found for $\mathrm{CoZr}$ and

TABLE I. Geometry of unreported prototypes (marked by * in Figs. 2-4). Atomic positions and unit-cell parameters are fully relaxed.

\begin{tabular}{|c|c|c|c|c|}
\hline System & $\mathrm{CoY}^{\star}$ & $\mathrm{Sc}_{2} \mathrm{Zr}^{\star}$ & $\begin{array}{l}\text { MoTi }^{\star} \\
\text { (Ref. 14) }\end{array}$ & $\begin{array}{l}\mathrm{Mo}_{3} \mathrm{Ti}^{\star} \\
\text { (Ref. 14) }\end{array}$ \\
\hline Lattice & Rhombohedral & Monoclinic & Orthorhombic & Orthorhombic \\
\hline Space group & $R \overline{3} m$ No. 166 & $C 2 / m$ No. 12 & Imma No. 74 & Immm No. 71 \\
\hline Pearson symbol & $\mathrm{hR} 4$ & $\mathrm{mS} 12$ & oI8 & oI8 \\
\hline Prim. vect. & (Hexagonal axes) & (SG option 1) & (SG option 2) & \\
\hline$a, b, c(\AA)$ & $3.896,3.896,20.609$ & $11.800,3.271,7.623$ & $4.479,3.182,9.046$ & $4.444,3.173,8.971$ \\
\hline$\alpha, \beta, \gamma(\mathrm{deg})$ & $90,90,120$ & $90,76.744,90$ & $90,90,90$ & $90,90,90$ \\
\hline \multirow{3}{*}{$\begin{array}{l}\text { Wyckoff positions } \\
\text { (Refs. } 42 \text { and } 43 \text { ) }\end{array}$} & Co1 $0,0,0.3326(6 c)$ & Sc1 $0.1914,0,-0.1370(4 i)$ & Mo1 0,1/4,0.3847 (4e) & Mo1 0,0,0.2440 (4i) \\
\hline & Y1 0,0,0.0869 (6c) & Sc2 $0.1334,0,0.4740(4 i)$ & Ti1 0,3/4, $-0.1343(4 e)$ & Mo2 0,1/2,0 (2d) \\
\hline & & $\mathrm{Zr} 1-0.4739,0,0.1931(4 i)$ & & Ti1 1/2,0,0 (2b) \\
\hline AFLOW label (Ref. 29) & “537” & “539” & “543” & “541” \\
\hline System & $\operatorname{ReTi}_{2}^{\star}$ & $\mathrm{Hf}_{2} \mathrm{Tl}^{\star}$ & $\mathrm{Be}_{2} \mathrm{Zn}^{\star}$ & $\mathrm{Re}_{3} \mathrm{Ru}^{\star}$ \\
\hline Lattice & Hexagonal & Tetragonal & Orthorhombic & Orthorhombic \\
\hline Space group & $P \overline{3} m 1$ No. 164 & I4/ $\mathrm{mmm}$ No. 139 & Fmmm No. 69 & Imm 2 No. 44 \\
\hline Pearson symbol & hP3 & tI6 & $\mathrm{oF} 12$ & oI8 \\
\hline Prim. vect. & (SG option 2) & (SG option 2) & (SG option 2) & \\
\hline$a, b, c(\AA)$ & $4.381,4.381,2.835$ & $4.422,4.422,7.385$ & $3.780,2.0978,10.300$ & $9.005,2.757,4.775$ \\
\hline$\alpha, \beta, \gamma(\operatorname{deg})$ & $90,90,120$ & $90,72.577,90$ & $90,90,90$ & $90,90,90$ \\
\hline Wyckoff positions & $\operatorname{Re} 10,0,0(1 a)$ & Hf1 0,0,0.1746 (4e) & Be1 0,0,0.17832 (8i) & $\operatorname{Re} 11 / 4,0,0(4 c)$ \\
\hline (Refs. 42 and 43) & Ti1 1/3,2/3,0.299 (2d) & T12 0,0,1/2 (2b) & Zn1 0,0,1/2 (4b) & $\begin{array}{c}\operatorname{Re} 20,1 / 2,1 / 6(2 b) \\
\operatorname{Ru} 10,0,2 / 3(2 a)\end{array}$ \\
\hline AFLOw label (Ref. 29) & “545” & “547" & “549” & “551” \\
\hline
\end{tabular}


CoHf, where the reported B2 structure is predicted to be replaced by degenerate B33/TlI structures, and for ReTi, where an unreported prototype, $\mathrm{MoTi}^{\star},{ }^{14}$ is predicted to replace $\mathrm{B} 2$ at low temperatures.

The structure map of the $A B_{2}$ compounds is shown in Fig. 3 . In this map there are eight predicted structures in reported phase-separating systems. Five of them appear in the Sc-Zr, $\mathrm{Hf}-\mathrm{Zr}$, Hf-Ti, Hf-Tl, and Be-Zn systems for which there are none in the $A B$ map (Fig. 2). The main features of the empirical map are maintained, which are a large cluster of C14 compounds and a smaller C15 group bordering it in the lower left part and a small $\mathrm{CuZr}_{2}$ group on the upper left. Discrepancies between reported and calculated structures appear at the margins of these clusters. In addition, small groups of C49 and C37 compounds are predicted in the upper part.

Figure 4 shows the structure map for the $A B_{3}$ compounds. Predicted structures appear in the Hf-Sc and Re-Tc systems, for which there are none in the $A B$ and $A B_{2}$ maps (Figs. 2 and 3 ). Thus, all the systems reported as phase separating but predicted to be compound forming have ordered structures in at least one of these three maps. In addition, a large cluster of 13 structures is predicted in the noncompound-forming gap in the center of the map. Eight of these are $\mathrm{D}_{19}$ structures and two have a closely related unreported hcp-prototype $\mathrm{Re}_{3} \mathrm{Ru}^{\star}$ (and OsRe $\mathrm{C}_{3}$ ). There are no major features in the $A B_{3}$ empirical map except a small group of D $0_{11}$ Y-compounds in the upper part. This group is reproduced and extended in the calculations. The reported prototype $\mathrm{Cd}_{3} \mathrm{Er}$, of $\mathrm{Cd}_{3} \mathrm{Y}$, is found to be degenerate with the $\mathrm{DO}_{19}$ structure.

Also predicted is a cluster of $\mathrm{Hf}$ and Ti compounds of the unreported prototype $\mathrm{Mo}_{3} \mathrm{Ti}^{\star} .{ }^{14}$ This concentration of distorted bcc structures $\left(\mathrm{Mo}_{3} \mathrm{Ti}^{\star}\right.$ is an oI8 distortion of a bcc superlattice, Table I) in six Hf and Ti binary systems may reflect their strong affinity to bcc environments. At zero temperature, bcc-Ti is dynamically unstable having an imaginary frequency phonon branch at the $\mathrm{N}$ point, ${ }^{40,41}$ but it stabilizes at $882{ }^{\circ} \mathrm{C}$, where Ti undergoes a hcp-bcc transition. ${ }^{34}$ Hence, an introduction of an alloying agent that stabilizes this phonon branch, can push the Ti alloy into a bcc-type environ- ment even at low temperature. Hf also undergoes an hcp-bcc transition at a much higher temperature of $1743{ }^{\circ} \mathrm{C} .{ }^{34}$ It is known, however, that alloying elements such as Os, Re, and Mo reduce this transition temperature and increase the stability domain of the bcc phase. ${ }^{35}$ The calculations show that a few alloying agents may be able to drive this extension down to very low temperatures.

\section{CONCLUSION}

Empirical structure maps employ correlations between simple properties of the elements (e.g., position in the periodic table) and known crystal types to predict stable ordered structures of new alloys. In contrast, $a b$ initio methods do not use previously available data but try to determine structure by computing from scratch a quantum-mechanical description of the system. The structure maps presented above merge the results of the empirical Pettifor maps and HT $a b$ initio calculations. The HT results reveal unsuspected clusters of structures both in binary systems known to be compound forming and in the empty gaps in the empirical maps, that are due to lack of experimental information. It is thus demonstrated that this integration of the two different approaches produces enhanced maps that should provide a more comprehensive improved foundation for rational materials design.

\section{ACKNOWLEDGMENTS}

We thank Wahyu Setyawan, Mike Mehl, Leeor Kronik, and Michal Jahnátek for fruitful discussions. This research was supported by ONR (Grants No. N00014-07-1-0878, No. N00014-07-1-1085, No. N00014-09-1-0921, and No. N00014-10-1-0436) and NSF (Grants No. DMR-0639822 and No. DMR-0650406). We are grateful for extensive use of the Fulton Supercomputer Center at Brigham Young University and Teragrid resources (MCA-07S005). S.C. acknowledges the Feinberg support at the Weizmann Institute of Science.

\footnotetext{
*Corresponding author; stefano@duke.edu

${ }^{1}$ J. Maddox, Nature (London) 335, 201 (1988).

${ }^{2}$ S. M. Woodley and R. Catlow, Nature Mater. 7, 937 (2008).

${ }^{3} \mathrm{P}$. Villars, in Crystal Structures of Intermetallic Compounds, edited by J. H. Westbrook and R. L. Fleisher (Wiley, New York, 1994), pp. 1-49.

${ }^{4}$ W. Hume-Rothery, The Metallic State (Oxford University Press, Oxford, 1931).

${ }^{5}$ A. R. Miedema, P. F. de Chatel, and F. R. de Boer, Physica B \& C 100, 1 (1980).

${ }^{6}$ A. Zunger, Phys. Rev. B 22, 5839 (1980).

${ }^{7}$ D. G. Pettifor, Solid State Commun. 51, 31 (1984).

${ }^{8}$ D. G. Pettifor, J. Phys. C 19, 285 (1986).

${ }^{9}$ D. G. Pettifor, J. Phys.: Condens. Matter 15, V13 (2003).

${ }^{10}$ P. Villars et al., J. Alloys Compd. 317-318, 26 (2001).
}

${ }^{11}$ G. H. Jóhannesson, T. Bligaard, A. V. Ruban, H. L. Skriver, K. W. Jacobsen, and J. K. Nørskov, Phys. Rev. Lett. 88, 255506 (2002).

${ }^{12}$ D. P. Stucke and V. H. Crespi, Nano Lett. 3, 1183 (2003).

${ }^{13}$ S. Curtarolo, D. Morgan, K. Persson, J. Rodgers, and G. Ceder, Phys. Rev. Lett. 91, 135503 (2003).

${ }^{14}$ S. Curtarolo, D. Morgan, and G. Ceder, CALPHAD: Comput. Coupling Phase Diagrams Thermochem. 29, 163 (2005).

${ }^{15}$ C. C. Fischer, K. J. Tibbetts, D. Morgan, and G. Ceder, Nature Mater. 5, 641 (2006).

${ }^{16}$ G. J. Lewis et al., J. Alloys Compd. 446-447, 355 (2007).

${ }^{17}$ V. Ozolins, E. H. Majzoub, and C. Wolverton, Phys. Rev. Lett. 100, 135501 (2008).

${ }^{18}$ O. Levy, R. V. Chepulskii, G. L. W. Hart, and S. Curtarolo, J. Am. Chem. Soc. 132, 833 (2010). 
${ }^{19}$ O. Levy, G. L. W. Hart, and S. Curtarolo, Acta Mater. 58, 2887 (2010).

${ }^{20}$ O. Levy, G. L. W. Hart, and S. Curtarolo, J. Am. Chem. Soc. 132, 4830 (2010).

${ }^{21}$ R. H. Taylor, S. Curtarolo, and G. L. W. Hart, Phys. Rev. B 81, 024112 (2010).

${ }^{22}$ J. M. Sanchez and D. de Fontaine, Phys. Rev. B 25, 1759 (1982).

${ }^{23}$ J. M. Sanchez, F. Ducastelle, and D. Gratias, Physica A 128, 334 (1984).

${ }^{24}$ R. P. McCormack, Ph.D. thesis, University of California, 1994.

${ }^{25}$ R. J. Lee and J. C. Raich, Phys. Rev. B 5, 1591 (1972).

${ }^{26}$ R. P. McCormack, M. Asta, G. Ceder, and D. de Fontaine, in Materials Theory and Modeling, MRS Symposia Proceedings No. 291 (Materials Research Society, Pittsburgh, 1993).

${ }^{27}$ M. Asta, R. P. McCormack, and D. de Fontaine, Phys. Rev. B 48, 748 (1993).

${ }^{28}$ A. van de Walle and G. Ceder, J. Phase Equilib. 23, 348 (2002).

${ }^{29}$ S. Curtarolo, G. L. W. Hart, W. Setyawan, R. V. Chepulskii, O. Levy, and D. Morgan, AFLOw: software for high-throughput calculation of material properties, http://materials.duke.edu/ aflow.html, 2009.

${ }^{30}$ G. Kresse and J. Hafner, Phys. Rev. B 47, 558 (1993).

${ }^{31}$ P. E. Blöchl, Phys. Rev. B 50, 17953 (1994).

${ }^{32}$ J. P. Perdew, K. Burke, and M. Ernzerhof, Phys. Rev. Lett. 77,
3865 (1996).

${ }^{33}$ H. J. Monkhorst and J. D. Pack, Phys. Rev. B 13, 5188 (1976).

${ }^{34}$ P. Villars et al., J. Alloys Compd. 367, 293 (2004).

${ }^{35}$ Binary Alloy Phase Diagrams, edited by T. B. Massalski, H. Okamoto, P. R. Subramanian, and L. Kacprzak (American Society for Metals, Materials Park, OH, 1990).

${ }^{36}$ G. L. W. Hart and R. W. Forcade, Phys. Rev. B 77, 224115 (2008).

${ }^{37}$ G. L. W. Hart and R. W. Forcade, Phys. Rev. B 80, 014120 (2009).

${ }^{38}$ A. N. Kolmogorov and S. Curtarolo, Phys. Rev. B 73, 180501(R) (2006).

${ }^{39}$ A. N. Kolmogorov and S. Curtarolo, Phys. Rev. B 74, 224507 (2006).

${ }^{40}$ W. Petry, A. Heiming, J. Trampenau, M. Alba, C. Herzig, H. R. Schober, and G. Vogl, Phys. Rev. B 43, 10933 (1991).

${ }^{41}$ S. R. Nishitani, H. Kawabe, and M. Aoki, Mater. Sci. Eng., A 312, 77 (2001).

${ }^{42}$ M. I. Aroyo, J. M. Perez-Mato, C. Capillas, E. Kroumova, S. Ivantchev, G. Madariaga, A. Kirov, and H. Wondratschek, Z. Kristallogr. 221, 15 (2006).

${ }^{43}$ Space Group Symmetry, International Tables of Crystallography Vol. A, edited by T. Hahn (Kluwer Academic, International Union of Crystallography, Chester, England, 2002). 